\title{
Análise epidemiológica da Sífilis Gestacional no estado de Minas Gerais
}

\author{
Epidemiological analysis of Gestational Syphilis in the state of Minas Gerais
}

Análisis epidemiológico de la sífilis gestacional en el estado de Minas Gerais

Stéphany Soares Santos ${ }^{1}$, Ana Luísa Braga Campos ${ }^{1}$, Ana Luísa Moreira Reis ${ }^{1}$, Andressa Ferreira Andrade $^{1 \star}$, Michelly Martins Nagai ${ }^{1}$, Samara Elisy Miranda Matos ${ }^{1}$, Marisa Costa e Peixoto ${ }^{1}$, Natália de Fátima Gonçalves Amâncio'.

\begin{abstract}
RESUMO
Objetivo: Explorar a epidemiologia da Sífilis Gestacional em Minas Gerais. Métodos: Refere-se de um estudo quantitativo, descritivo e transversal, executado em Minas Gerais (MG), de 2010 a 2019. A coleção de dados foi feita por meio do Sistema de Informação de Agravos de Notificação SINAN - DATASUS, considerando os casos confirmados de Sífilis Gestacional. As variáveis consideradas foram faixa etária, raça/cor, escolaridade, ano do diagnóstico, idade gestacional, classificação clínica e esquema de tratamento. Resultados: É possível constatar, por meio desse estudo, maior incidência em mulheres de 20 a 29 anos, de raça/cor parda, com fundamental incompleto, idade gestacional do diagnóstico no terceiro trimestre, tendo como forma mais prevalente a sífilis latente e tratamento realizado com penicilina. Conclusão: Observa-se aumento na incidência da Sífilis na Gestação no estado de Minas Gerais, com o perfil de mulheres adultas jovens, pardas e que têm ensino fundamental incompleto. O diagnóstico ocorre forma tardia, no terceiro trimestre, e o tratamento, na maioria dos casos, é feito com penicilina. A análise epidemiológica foi feita através de informações disponíveis nas fichas de notificações, isso reforça a importância e a necessidade de manter os dados atualizados.
\end{abstract}

Palavras-chave: Sífilis, Epidemiologia, Complicações infecciosas na gravidez.

\begin{abstract}
Objective: To explore the epidemiology of Gestational Syphilis in Minas Gerais. Methods: Refers to a quantitative, descriptive and cross-sectional study, carried out in Minas Gerais (MG), from 2010 to 2019. The data collection was done through the SINAN - DATASUS Notifiable Diseases Information System, considering the cases confirmed gestational syphilis. The variables considered were age, race/color, education, year of diagnosis, gestational age, clinical classification and treatment scheme. Results: It is possible to verify, through this study, a higher incidence in women of 20 to 29 years of age, of mixed race/color, with incomplete primary education, gestational age of diagnosis in the third trimester, with latent syphilis as the most prevalent form and treatment performed with penicillin. Conclusion: There is an increase in the incidence of syphilis in pregnancy in the state of Minas Gerais, with the profile of young adult women, brown and who have incomplete elementary education. The diagnosis occurs late in the third trimester, and treatment, in most cases, is done with penicillin. The epidemiological analysis was done through information available in the notification forms, this reinforces the importance and the need to keep the data up to date.
\end{abstract}

Key words: Syphilis, Epidemiology, Pregnancy complications infectious.

\section{RESUMEN}

Objetivo: Explorar la epidemiología de la sífilis gestacional en Minas Gerais. Métodos: Se refiere a un estudio cuantitativo, descriptivo y transversal, realizado en Minas Gerais (MG), de 2010 a 2019. La recolección de datos se realizó a través del Sistema de Información de Enfermedades Notificables SINAN - DATASUS, considerando los casos confirmados de sífilis gestacional. Las variables consideradas fueron edad, raza/color, educación, año de diagnóstico, edad gestacional, clasificación clínica y esquema de tratamiento. Resultados: Es posible verificar, a través de este estudio, una mayor incidencia en mujeres de 20 a 29 años, mestizos, con educación primaria incompleta, edad gestacional de diagnóstico en el tercer trimestre, con sífilis latente

${ }^{1}$ Centro Universitário de Patos de Minas (UNIPAM), Patos de Minas - MG.

*E-mail: andressafa@unipam.edu.br

SUBMETIDO EM: 2/2021

ACEITO EM: 3/2021

PUBLICADO EM: $3 / 2021$ 
como forma más prevalente y tratamiento realizado con penicilina. Conclusión: Existe un aumento en la incidencia de sífilis en el embarazo en el estado de Minas Gerais, con el perfil de mujeres adultas jóvenes, morenas y que tienen educación primaria incompleta. El diagnóstico se produce al final del tercer trimestre y el tratamiento, en la mayoría de los casos, se realiza con penicilina. El análisis epidemiológico se realizó utilizando la información disponible en los formularios de notificación, lo que refuerza la importancia y la necesidad de mantener los datos actualizados.

Palabras clave: Sífilis, Epidemiología, Complicaciones infecciosas del embarazo

\section{INTRODUÇÃO}

A sífilis é uma doença infectocontagiosa sistêmica, causada pela espiroqueta da bactéria gram-negativa Treponema pallidum, cujo habitat no organismo é, geralmente, a mucosa urogenital (JÚNIOR WVO, et al., 2020). É transmitida através de via sexual (sífilis adquirida) e também vertical (sífilis congênita), pela placenta da mãe para o feto ou por contato direto com a lesão no momento do parto (GOMES NS, et al., 2020).

A forma adquirida se divide em recente e tardia depende do tempo de infecção e do grau de infectividade. A recente manifesta-se em menos de 2 anos após o contato e é subdividida em 3 estágios: primário, secundário e latente recente. A primária é caracterizada, principalmente, pela manifestação de cancro duro, que pode estar acompanhado de linfoadenomegalia regional. Na secundária, são comuns sinais e sintomas sistêmicos, erupções cutâneas e lesões eritemato-escamosas palmo-plantares, tornando as lesões na pele e nas mucosas mais evidentes (GOMES NS, et al., 2020).

No estágio de latência, não se observam manifestações clínicas, mas os testes com anticorpos são reagentes (o estágio latente recente ocorre nos primeiros 2 anos de infecção, e o tardio após esse período). Essa fase pode ser interrompida pelo surgimento de sinais e sintomas da forma secundária ou terciária; sendo que na terciária, pode ocorrer o acometimento do sistema nervoso e cardiovascular. A fase se latência tardia e a sífilis terciária são características da sífilis tardia (ARAÚJO AGR, et al., 2018).

Essa patologia pode ser influenciada por alguns marcadores socioeconômicos e questões epidemiológicas. Conforme apontado em uma pesquisa de campo por Cândido DS, et al. (2019), a sífilis tem uma incidência elevada e crescente em indivíduos do sexo masculino. Tal realidade está diretamente relacionada ao fato de o homem frequentemente negligenciar o cuidado com a saúde. Segundo o mesmo autor, em períodos festivos os casos tendem aumentar, principalmente entre os jovens. Isso deve-se ao fato de que nesses períodos, os indivíduos dessa faixa etária tendem a ter mais relações sexuais casuais desprotegidas. Nesse contexto, a promiscuidade entre os sexos e a falta de proteção nas relações sexuais contribuem significativamente para que o contágio seja cada vez mais elevado (CÂNDIDO DS, et al., 2019).

A triagem de sífilis faz parte do exame pré-natal e deve ser realizada no $1^{\circ}$ trimestre (preferencialmente na primeira consulta pré-natal), no $3^{\circ}$ trimestre de gestação por volta da $30^{\text {a }}$ semana de gestação e no momento da internação hospitalar, para parto ou curetagem uterina por aborto (BRASIL, 2018). Caso a sífilis seja detectada, é necessária intervenção imediata, reduzindo, assim, a possibilidade de transmissão vertical (ARAÚJO AGR, et al., 2018). Em gestantes infectadas não tratadas ou tratadas inadequadamente, a chance de transmissão da doença para o feto é de 70 a 100\%. Essa transmissão pode ocorrer em qualquer momento da gestação ou do estágio clínico da doença e pode ter diversos desfechos desfavoráveis como o abortamento, o natimorto, a prematuridade e as malformações fetais (CRUZ VC, 2019).

Apesar de apresentar diagnóstico simples e tratamento eficaz, a sífilis gestacional permanece com prevalência alarmante, especialmente em países subdesenvolvidos ou em desenvolvimento (GOMES NS, et al., 2020). Nesse contexto, a assistência prestada a essa população tem como objetivo reduzir o risco materno, fetal e do neonato, de acordo com o recomendado pela Política Nacional de Atenção à Saúde Integral da Mulher (CABRAL BTV, et al., 2017).

No Brasil, a Atenção Básica (AB) é a responsável por promover ações voltadas para saúde, incluindo o apoio gestacional (BRASIL, 2017). Como a sífilis é considerada uma afecção suscetível de controle, e a AB responsável pela assistência à saúde da mulher; entende-se que toda a equipe deve planejar ações de prevenção, promoção e assistência a gestante com sífilis (CABRAL BTV, et al., 2017). 
Dado o aumento considerável da incidência e prevalência de sífilis, a importância da análise epidemiológica na construção de ações estratégicas se faz fundamental. Considera-se pertinente analisar o perfil epidemiológico da doença de maneira regional, com intuito de avaliar o seu controle no todo. Diante disso, é de fundamental importância o aprimoramento de estratégias e o fortalecimento dos órgãos prestadores de serviços, incluindo a notificação compulsória, pois, os dados são indispensáveis para avaliação da vigilância epidemiológica e para os gestores planejarem intervenções eficientes (CABRAL BTV, et al., 2017).

O estudo tem por objetivo analisar a incidência de sífilis gestacional e congênita a partir de dados obtidos no DATASUS, no estado de Minas Gerais, a fim de criar aporte para o estabelecimento de medidas eficazes.

\section{MÉTODOS}

Esta pesquisa se classifica como um estudo quantitativo, descritivo, do tipo transversal. Realizou-se o levantamento dos casos confirmados de Sífilis na gestação, registrados no Sistema de Informação de Agravos e Notificação - SINAN - DATASUS, no estado de Minas Gerais. Consideraram-se casos no período de 2010 a 2019, sendo estudadas as seguintes variáveis: faixa etária, sexo, raça/cor, escolaridade, ano do diagnóstico, idade gestacional, classificação clínica e esquema de tratamento. Foram incluídos dados epidemiológicos sobre sífilis gestacional no estado de Minas Gerais no período de 2010 a 2019 publicados no Datasus e foram excluídos dados da sífilis gestacional referentes ao mesmo período no estado de Minas Gerais não divulgados no Datasus.

Posteriormente, os dados foram designados em uma tabela no Microsoft Excel, para a elaboração das tabelas presentes ao longo do artigo, que apresentam frequência e distribuição dos casos. Calcularam-se as taxas de incidência desta patologia durante a gestação na população estudada, por ano de diagnóstico. Não foi necessária a apreciação do Comitê de Ética em Pesquisa - CEP pelo fato de se tratar de dados públicos. O trabalho foi realizado nas seguintes etapas: (I) pesquisa bibliográfica, (II) análise e coleta de dados a respeito dos casos de sífilis na gestação no estado de Minas Gerais no período estudado (III) e descrição dos dados encontrados.

\section{RESULTADOS E DISCUSSÃO}

Durante os anos de 2010 a 2019, as gestantes com idades entre 20 e 29 anos apresentaram maiores taxas de incidência de sífilis, com o percentual médio de casos nesta faixa etária de $52,8 \%$, variando de $49 \%$ a $56,1 \%$ ao longo dos anos (Tabela 1 ).

Tabela 1 - Distribuição absoluta (n) e percentual (\%) de casos de gestantes com sífilis segundo faixa etária por ano de diagnóstico.

\begin{tabular}{cccccc}
\hline Ano & $\begin{array}{c}\mathbf{1 0} \text { a 14 anos } \mathbf{n} \\
(\%)\end{array}$ & $\begin{array}{c}\mathbf{1 5} \text { a 19 anos } \mathbf{n} \\
(\mathbf{\%})\end{array}$ & $\begin{array}{c}\mathbf{2 0} \text { a 29 anos } \mathbf{n} \\
(\mathbf{\%})\end{array}$ & $\begin{array}{c}\mathbf{3 0} \text { a } \mathbf{3 9} \text { anos } \mathbf{n} \\
(\%)\end{array}$ & $\begin{array}{c}\mathbf{4 0} \text { anos ou mais } \\
\mathbf{n}(\%)\end{array}$ \\
\hline $\mathbf{2 0 1 0}$ & $4(1,1)$ & $71(19,6)$ & $203(56,1)$ & $77(21,3)$ & $7(1,9)$ \\
$\mathbf{2 0 1 1}$ & $10(1,8)$ & $128(23,3)$ & $270(49,1)$ & $118(21,5)$ & $24(4,4)$ \\
$\mathbf{2 0 1 2}$ & $9(1)$ & $201(23,1)$ & $404(46,4)$ & $238(27,3)$ & $19(2,2)$ \\
$\mathbf{2 0 1 3}$ & $17(1,5)$ & $299(26,3)$ & $590(51,9)$ & $214(18,8)$ & $17(1,5)$ \\
$\mathbf{2 0 1 4}$ & $27(1,6)$ & $486(28,6)$ & $832(49)$ & $320(18,8)$ & $33(1,9)$ \\
$\mathbf{2 0 1 5}$ & $28(1,2)$ & $632(26,2)$ & $1247(51,7)$ & $460(19,1)$ & $43(1,8)$ \\
$\mathbf{2 0 1 6}$ & $33(1,3)$ & $656(25,2)$ & $1403(53,9)$ & $474(18,2)$ & $39(1,5)$ \\
$\mathbf{2 0 1 7}$ & $34(0,9)$ & $1000(27,3)$ & $1936(52,8)$ & $625(17)$ & $72(2)$ \\
$\mathbf{2 0 1 8}$ & $47(1)$ & $1217(25,1)$ & $2637(54,4)$ & $865(17,8)$ & $85(1,8)$ \\
$\mathbf{2 0 1 9}$ & $19(1)$ & $440(23,3)$ & $1062(56,2)$ & $331(17,5)$ & $38(2)$ \\
Total & $228(1,1)$ & $5130(25,6)$ & $10584(52,8)$ & $3722(18,6)$ & $377(1,9)$
\end{tabular}

Notas: (1) Dados até 30/06/2019; (2) Dados preliminares para os últimos 5 anos.

Fonte: Santos SS, et al., 2021; dados extraídos de MS/SVS/DCCI - Departamento de Doenças de Condições Crônicas e Infecções Sexualmente Transmissíveis. 
Outros estudos brasileiros atuais mostram resultados semelhantes. Um estudo realizado no município de Divinópolis - MG, o qual teve como objetivo fazer a análise do perfil epidemiológico da sífilis em mulheres entre os anos de 2011 a 2016, mostrou que a faixa etária mais acometida em gestantes é também de 20 a 29 anos, com uma taxa de ocorrência média da doença de 53,5\% (ANDRADE HS, et al., 2019). Similarmente, outro estudo realizado no município de Montes Claros - MG evidenciou que a faixa etária de 20 a 29 anos é mais afetada pelos casos de sífilis na gestação (ARAÚJO AGR, et al., 2018).

A segunda faixa etária mais atingida pela doença no presente estudo foi de 15 a 19 anos e também está de acordo com dados de outros estudos brasileiros (ARAÚJO AGR, et al., 2018; MACHIO-LIMA T, et al., 2019; SANTOS VF, et al., 2019). De acordo com alguns pesquisadores, a elevada ocorrência de casos de sífilis em mulheres em idade jovem, de 15 a 29 anos, pode estar relacionada ao fato de estas mulheres possuírem vida sexual mais ativa (MACHIO-LIMA T, et al., 2019). Pode-se também notar que as taxas de incidência de sífilis, na faixa etária mais acometida das gestantes (20 a 29 anos), apresentaram-se de forma crescente nos últimos três anos analisados, correspondendo a 52,8\% em 2017, 54,4\% em 2018 e 56,2\% em 2019.

Os incrementos nas taxas de incidência da doença observados podem ser resultado do aumento da realização de notificações em consequência da intensificação da vigilância sanitária relacionada à sífilis. Além disso, podem estar associados ao acompanhamento pré-natal que vem ocorrendo de forma mais eficaz, proporcionando a realização de mais testes rápidos para detecção de sífilis na gestação (ANDRADE HS, et al., 2019). A maior parte dos diagnósticos de sífilis na gestação se deu entre mulheres que se autodeclararam como pardas durante os anos de 2010 a 2019, representando uma taxa média de $48,1 \%$ dos casos da doença, variando $34,5 \%$ a $54,7 \%$ ao longo dos anos.

Pode-se observar, também, que a porcentagem de mulheres cuja raça ou cor era ignorada, ou seja, desconhecida, foi diminuindo ao longo dos anos, passando de 19,1\% em 2010 para 3,3\% em 2019 e, paralelamente, a porcentagem de mulheres pardas acometidas pela doença foi aumentando ao longo dos anos, passando de $34,5 \%$ em 2010 para 48,1\% em 2019. Tais dados podem ser reflexo de uma melhor percepção da mulher sobre sua própria cor ou etnia nos últimos anos e/ou ainda podem estar associados a uma melhoria na forma de preenchimento das fichas de notificação da doença (Tabela 2).

Tabela 2 - Distribuição absoluta (n) e percentual (\%) de casos de gestantes com sífilis segundo raça ou cor por ano de diagnóstico.

\begin{tabular}{ccccccc}
\hline Ano & $\begin{array}{c}\text { Branca } \\
\mathbf{n}(\%)\end{array}$ & $\begin{array}{c}\text { Preta } \\
\mathbf{n}(\%)\end{array}$ & $\begin{array}{c}\text { Amarela } \\
\mathbf{n}(\%)\end{array}$ & $\begin{array}{c}\text { Parda } \\
\mathbf{n}(\%)\end{array}$ & $\begin{array}{c}\text { Indígena } \\
\mathbf{n}(\%)\end{array}$ & $\begin{array}{c}\text { Ignorada } \\
\mathbf{n}(\%)\end{array}$ \\
\hline $\mathbf{2 0 1 0}$ & $96(26,5)$ & $66(18,2)$ & $6(1,7)$ & $125(34,5)$ & 0 & $69(19,1)$ \\
$\mathbf{2 0 1 1}$ & $134(24,4)$ & $104(18,9)$ & $9(1,6)$ & $210(38,2)$ & 0 & $93(16,9)$ \\
$\mathbf{2 0 1 2}$ & $216(24,8)$ & $173(19,9)$ & $7(0,8)$ & $324(37,2)$ & $4(0,5)$ & $147(16,9)$ \\
$\mathbf{2 0 1 3}$ & $269(23,6)$ & $167(14,7)$ & $10(0,9)$ & $462(40,6)$ & $2(0,2)$ & $228(20)$ \\
$\mathbf{2 0 1 4}$ & $390(23)$ & $262(15,4)$ & $14(0,8)$ & $707(41,6)$ & 0 & $325(19,1)$ \\
$\mathbf{2 0 1 5}$ & $544(22,6)$ & $352(14,6)$ & $26(1,1)$ & $1061(44)$ & $5(0,2)$ & $422(17,5)$ \\
$\mathbf{2 0 1 6}$ & $583(22,4)$ & $370(14,2)$ & $31(1,2)$ & $1217(46,7)$ & $2(0,1)$ & $402(15,4)$ \\
$\mathbf{2 0 1 7}$ & $912(24,9)$ & $569(15,5)$ & $42(1,1)$ & $1902(51,9)$ & $1(0,2)$ & $241(6,6)$ \\
$\mathbf{2 0 1 8}$ & $1133(23,4)$ & $792(16,3)$ & $55(1,1)$ & $2610(53,8)$ & $9(0,2)$ & $252(5,2)$ \\
$\mathbf{2 0 1 9}$ & $455(24,1)$ & $312(16,5)$ & $24(1,3)$ & $1034(54,7)$ & $2(0,1)$ & $63(3,3)$ \\
\hline Total & $4732(23,6)$ & $3167(15,8)$ & $224(1,1)$ & $9652(48,1)$ & $25(0,1)$ & $2242(11,1)$ \\
\hline
\end{tabular}

Notas: (1) Dados até 30/06/2019; (2) Dados preliminares para os últimos 5 anos.

Fonte: Santos SS, et al., 2021; dados extraídos de MS/SVS/DCCI - Departamento de Doenças de Condições Crônicas e Infecções Sexualmente Transmissíveis. 
Outros estudos brasileiros realizados em outras regiões apresentam resultados semelhantes em relação à raça ou cor das gestantes com sífilis. Um estudo realizado na região sul do país com o intuito de analisar a prevalência de sífilis na gestação e sua associação com características socioeconômicas, histórico reprodutivo, assistência no pré-natal e no parto mostrou que a ocorrência da sífilis na gestação esteve associada à raça/cor não branca, o que sugere que a ocorrência da doença em mulheres pardas também é elevada (PADOVANI C, et al., 2018).

Da mesma maneira, um estudo conduzido na região nordeste revelou que os números de diagnósticos de sífilis em gestantes de cor parda ao longo dos anos de 2014 a 2019 sempre foram mais elevados do que aqueles de gestantes brancas ou negras (BARBOSA DFR, et al., 2020).

Analogamente, um trabalho desenvolvido na região norte para descrever o perfil epidemiológico da sífilis gestacional e congênita entre os anos de 2015 a 2017 evidenciou que mais de $90 \%$ das gestantes com sífilis eram pardas (SABACK MC, et al., 2018). É importante destacar que a alta prevalência de indivíduos pardos na população brasileira pode ter contribuído para os resultados encontrados.

É possível observar uma relação inversamente proporcional entre o nível de instrução e diagnóstico de sífilis na gestação. Como exemplo, observa-se que mães que são analfabetas ou apresentam ensino fundamental incompleto somam 4515 casos, no período de 2010 ao primeiro semestre de 2019, contabilizando um percentil de 22,9\%. Além disso, é possível generalizar, que no ano de 2018, com exceção ao analfabetismo, os diagnósticos de todos os níveis de instrução sofreram um aumento considerável. Temos como exemplo as confirmações de sífilis no ensino médio completo, que em 2010 apresentava 45 casos e, em 2018, 897 diagnósticos (Tabela 3).

Tabela 3 - Distribuição absoluta (n) e percentual (\%) de casos de gestantes com sífilis segundo escolaridade por ano de diagnóstico.

\begin{tabular}{|c|c|c|c|c|c|c|c|c|c|c|c|}
\hline $\begin{array}{l}\text { Nível de } \\
\text { Instrução }\end{array}$ & $\begin{array}{l}2010 \\
\text { n (\%) }\end{array}$ & $\begin{array}{l}2011 \\
\text { n (\%) }\end{array}$ & $\begin{array}{l}2012 \\
\text { n (\%) }\end{array}$ & $\begin{array}{l}2013 \\
\text { n (\%) }\end{array}$ & $\begin{array}{l}2014 \\
\text { n (\%) }\end{array}$ & $\begin{array}{l}2015 \\
\text { n (\%) }\end{array}$ & $\begin{array}{l}2016 \\
\text { n (\%) }\end{array}$ & $\begin{array}{l}2017 \\
\text { n (\%) }\end{array}$ & $\begin{array}{l}2018 \\
\text { n (\%) }\end{array}$ & $\begin{array}{l}2019 \\
\text { n (\%) }\end{array}$ & $\begin{array}{l}\text { Total } \\
\text { n (\%) }\end{array}$ \\
\hline Analfabeto & $\begin{array}{c}2 \\
(0,6)\end{array}$ & $\begin{array}{c}2 \\
(0,4)\end{array}$ & $\begin{array}{c}6 \\
(0,7)\end{array}$ & $\begin{array}{c}4 \\
(0,4)\end{array}$ & $\begin{array}{c}8 \\
(0,5)\end{array}$ & $\begin{array}{c}9 \\
(0,4)\end{array}$ & $\begin{array}{c}7 \\
(0,3)\end{array}$ & $\begin{array}{c}12 \\
(0,3)\end{array}$ & $\begin{array}{c}10 \\
(0,2)\end{array}$ & $\begin{array}{c}6 \\
(0,3)\end{array}$ & $\begin{array}{c}66 \\
(0,3)\end{array}$ \\
\hline $\begin{array}{l}\text { Fundamental } \\
\text { Incompleto }\end{array}$ & $\begin{array}{c}99 \\
(26,5)\end{array}$ & $\begin{array}{c}146 \\
(26,6)\end{array}$ & $\begin{array}{l}218 \\
(25)\end{array}$ & $\begin{array}{l}273 \\
(24)\end{array}$ & $\begin{array}{c}369 \\
(21,8)\end{array}$ & $\begin{array}{c}502 \\
(20,8)\end{array}$ & $\begin{array}{c}501 \\
(19,2)\end{array}$ & $\begin{array}{c}847 \\
(23,1)\end{array}$ & $\begin{array}{c}1093 \\
(22,5)\end{array}$ & $\begin{array}{c}404 \\
(21,3)\end{array}$ & $\begin{array}{l}4449 \\
(22,6)\end{array}$ \\
\hline $\begin{array}{l}\text { Fundamental } \\
\text { Completo }\end{array}$ & $\begin{array}{c}35 \\
(9,7)\end{array}$ & $\begin{array}{c}40 \\
(7,3)\end{array}$ & $\begin{array}{c}71 \\
(8,2)\end{array}$ & $\begin{array}{c}84 \\
(7,4)\end{array}$ & $\begin{array}{c}134 \\
(7,9)\end{array}$ & $\begin{array}{l}215 \\
(8,9)\end{array}$ & $\begin{array}{l}221 \\
(8,5)\end{array}$ & $\begin{array}{c}385 \\
(10,5)\end{array}$ & $\begin{array}{c}526 \\
(10,8)\end{array}$ & $\begin{array}{c}225 \\
(11,9)\end{array}$ & $\begin{array}{l}1936 \\
(9,6)\end{array}$ \\
\hline $\begin{array}{c}\text { Médio } \\
\text { Incompleto }\end{array}$ & $\begin{array}{c}36 \\
(9,9)\end{array}$ & $\begin{array}{c}45 \\
(8,2)\end{array}$ & $\begin{array}{c}84 \\
(9,6)\end{array}$ & $\begin{array}{l}113 \\
(9,9)\end{array}$ & $\begin{array}{c}176 \\
(10,4)\end{array}$ & $\begin{array}{c}263 \\
(10,9)\end{array}$ & $\begin{array}{c}301 \\
(11,6)\end{array}$ & $\begin{array}{c}529 \\
(14,4)\end{array}$ & $\begin{array}{c}692 \\
(14,3)\end{array}$ & $\begin{array}{c}267 \\
(14,1)\end{array}$ & $\begin{array}{l}2506 \\
(12,3)\end{array}$ \\
\hline $\begin{array}{l}\text { Médio } \\
\text { Completo }\end{array}$ & $\begin{array}{c}45 \\
(12,4)\end{array}$ & $\begin{array}{c}70 \\
(12,7)\end{array}$ & $\begin{array}{c}85 \\
(9,8)\end{array}$ & $\begin{array}{c}98 \\
(8,6)\end{array}$ & $\begin{array}{l}203 \\
(12)\end{array}$ & $\begin{array}{c}279 \\
(11,6)\end{array}$ & $\begin{array}{c}310 \\
(11,9)\end{array}$ & $\begin{array}{c}612 \\
(16,7)\end{array}$ & $\begin{array}{c}897 \\
(18,5)\end{array}$ & $\begin{array}{c}407 \\
(21,5)\end{array}$ & $\begin{array}{r}3006 \\
(14,7)\end{array}$ \\
\hline $\begin{array}{l}\text { Superior } \\
\text { Incompleto }\end{array}$ & $\begin{array}{c}1 \\
(0,3)\end{array}$ & $\begin{array}{c}3 \\
(0,5)\end{array}$ & $\begin{array}{c}7 \\
(0,8)\end{array}$ & $\begin{array}{c}8 \\
(0,7)\end{array}$ & $\begin{array}{c}11 \\
(0,6)\end{array}$ & $\begin{array}{c}27 \\
(1,1)\end{array}$ & $\begin{array}{c}19 \\
(0,7)\end{array}$ & $\begin{array}{c}32 \\
(0,9)\end{array}$ & $\begin{array}{c}65 \\
(1,3)\end{array}$ & $\begin{array}{c}22 \\
(1,2)\end{array}$ & $\begin{array}{l}195 \\
(0,9)\end{array}$ \\
\hline $\begin{array}{l}\text { Superior } \\
\text { Completo }\end{array}$ & $\begin{array}{c}3 \\
(0,8)\end{array}$ & - & $\begin{array}{c}4 \\
(0,5)\end{array}$ & $\begin{array}{c}5 \\
(0,4)\end{array}$ & $\begin{array}{c}10 \\
(0,6)\end{array}$ & $\begin{array}{c}17 \\
(0,7)\end{array}$ & $\begin{array}{c}19 \\
(0,7)\end{array}$ & $\begin{array}{c}31 \\
(0,8)\end{array}$ & $\begin{array}{l}50 \\
(1)\end{array}$ & $\begin{array}{l}18 \\
(1)\end{array}$ & $\begin{array}{c}157 \\
(0,8)\end{array}$ \\
\hline Não se aplica & - & - & - & $\begin{array}{c}1 \\
(0,1)\end{array}$ & - & - & - & - & - & - & $\begin{array}{c}1 \\
(0)\end{array}$ \\
\hline Ignorado & $\begin{array}{c}144 \\
(39,8)\end{array}$ & $\begin{array}{c}244 \\
(44,4)\end{array}$ & $\begin{array}{c}396 \\
(45,5)\end{array}$ & $\begin{array}{c}552 \\
(48,5)\end{array}$ & $\begin{array}{c}787 \\
(46,3)\end{array}$ & $\begin{array}{c}1098 \\
(45,6)\end{array}$ & $\begin{array}{c}1227 \\
(47,1)\end{array}$ & $\begin{array}{c}1219 \\
(33,2)\end{array}$ & $\begin{array}{c}1518 \\
(31,3)\end{array}$ & $\begin{array}{c}541 \\
(28,6)\end{array}$ & $\begin{array}{l}7726 \\
(38,8)\end{array}$ \\
\hline
\end{tabular}

Notas: (1) Dados até 30/06/2019; (2) Dados preliminares para os últimos 5 anos.

Fonte: Santos SS, et al., 2021; dados extraídos de MS/SVS/DCCI - Departamento de Doenças de Condições Crônicas e Infecções Sexualmente Transmissíveis. 
Em um estudo semelhante realizado em Juiz de Fora - MG, no período entre 2010 e 2017, concluiu-se que o baixo nível de escolaridade influencia na falta de informação sobre uso de preservativo na gestação, bem como as possíveis consequências para o concepto, destacando-se, assim, na estatística do diagnóstico de sífilis em gestantes (PEREIRA AL, et al., 2020). Paralelamente, em um estudo epidemiológico realizado no Rio de Janeiro, entre 2009 e 2018, foi encontrada uma prevalência dos diagnósticos em gestantes de menor grau de instrução, somando 7255 casos apresentados em pacientes analfabetas ou com ensino médio incompleto.

Os dados analisados destacam a vulnerabilidade e a informalidade deste grupo, expondo a necessidade de novas medidas de promoção da saúde, que abrangem a simplificação da educação sexual bem como da saúde íntima feminina e por fim abrangendo o pré-natal, com o objetivo de aumentar o amparo das mães e de seus filhos. (CÂMARA LS, et al., 2020). Analisando o aumento expressivo de diagnósticos recentes, independentemente do nível de instrução, pode-se concluir a ineficácia da cobertura do sistema de pré-natal, tendo como barreira a falta de acessibilidade a informação de forma inteligível. Por muitas vezes a ineficiência está no descumprimento de protocolos de consulta, ginecológicas e obstétricas, não ocorrendo a solicitação de exames e a orientação quanto aos riscos e prevenção da sífilis (MACÊDO VC, et al., 2020).

Os dados desta discussão se assemelham a uma análise comparativa, do boletim epidemiológico publicado pelo Ministério da Saúde, em que a Sífilis se encontra como uma doença de uma parcela marginalizada, caracterizada pelo baixo nível de escolaridade e socioeconômico. Sendo assim, o estudo destaca a importância no desenvolvimento de políticas de saúde pública que ofereçam a equidade necessária para que estes grupos sejam amparados (MORAIS TR, et al., 2019). Um estudo realizado no estado de Goiás, sobre o perfil de casos notificados de sífilis congênita entre 2015 a 2018, destacou a importância da expansão do atendimento pré-natal com a adesão da rede cegonha, que prioriza a assistência materno-infantil, afim de oferecer e ampliar a promoção da saúde (SOUZA RR, et al., 2020).

A respeito da relação da idade gestacional e a ocorrência de casos de sífilis é possível destacar o $3^{\circ}$ trimestre de 2018, como sendo o de maior prevalência em diagnósticos, apresentando 1794 casos, além de somar, durante o período de 2010 a 2019, 7877 confirmações. Além disso, tem-se em evidência um elevado número de diagnósticos no ano de 2018, independente do trimestre. É possível comparar este crescimento com relação ao ano de 2010 que apresentava 60 casos no primeiro, 114 no segundo e 166 no terceiro trimestre. Já em 2018 estes números se apresentaram por 1601, 1113 e 1794 casos respectivamente (Tabela 4).

Tabela 4 - Distribuição absoluta (n) e percentual (\%) de casos de gestantes com sífilis segundo idade gestacional por ano de diagnóstico.

\begin{tabular}{ccccc}
\hline Ano & $\begin{array}{c}\mathbf{1}^{\circ} \text { Trimestre } \\
\mathbf{n}(\%)\end{array}$ & $\begin{array}{c}\mathbf{2}^{\circ} \text { Trimestre } \\
\mathbf{n}(\%)\end{array}$ & $\begin{array}{c}\mathbf{3}^{\circ} \text { Trimestre } \\
\mathbf{n}(\%)\end{array}$ & $\begin{array}{c}\text { Idade gestacional } \\
\text { ignorada } \\
\mathbf{n}(\%)\end{array}$ \\
\hline $\mathbf{2 0 1 0}$ & $60(27,4)$ & $114(25,4)$ & $166(45,9)$ & $22(6,1)$ \\
$\mathbf{2 0 1 1}$ & $111(20,2)$ & $171(31,1)$ & $218(39,6)$ & $50(9,1)$ \\
$\mathbf{2 0 1 2}$ & $193(22,2)$ & $245(28,1)$ & $364(41,8)$ & $69(7,9)$ \\
$\mathbf{2 0 1 3}$ & $264(23,2)$ & $305(26,8)$ & $465(40,9)$ & $104(9,1)$ \\
$\mathbf{2 0 1 4}$ & $373(22)$ & $420(24,7)$ & $731(43,1)$ & $174(10,2)$ \\
$\mathbf{2 0 1 5}$ & $534(22,2)$ & $603(25)$ & $1048(43,5)$ & $225(9,3)$ \\
$\mathbf{2 0 1 6}$ & $657(25,2)$ & $675(25,9)$ & $1065(40,9)$ & $208(8)$ \\
$\mathbf{2 0 1 7}$ & $1147(31,3)$ & $922(25,1)$ & $1366(37,3)$ & $232(6,3)$ \\
$\mathbf{2 0 1 8}$ & $1601(33)$ & $1113(22,9)$ & $1794(37)$ & $343(7,1)$ \\
$\mathbf{2 0 1 9}$ & $640(33,9)$ & $491(26)$ & $660(34,9)$ & $99(5,2)$ \\
\hline Total & $5580(27,4)$ & $5059(25,4)$ & $7877(39,5)$ & $1526(7,7)$ \\
\hline
\end{tabular}

Notas: (1) Dados até 30/06/2019; (2) Dados preliminares para os últimos 5 anos.

Fonte: Santos SS, et al., 2021; dados extraídos de MS/SVS/DCCI - Departamento de Doenças de Condições Crônicas e Infecções Sexualmente Transmissíveis. 
Os dados do terceiro trimestre no maior número de diagnósticos se tornam bastante alarmante, pois expressa um serviço de assistência baixo, tendo como consequência uma alta demanda de busca para o prénatal de forma tardia, demostrando ineficiência nos projetos de educação da saúde básica, evidenciando assim uma necessidade de reformulação da estrutura e disseminação destes programas (CONCEIÇÃO HN, et al., 2019).

Em análise, o crescente número de diagnóstico recentes de sífilis gestacional encontra-se em consonância com uma pesquisa retrospectiva realizada em Caxias - MA, no período de 2012 a 2017, que constatou esta mesma evolução, concluindo, assim, sobre a inefetividade na disseminação de informações de prevenção, porém paradoxalmente com um maior alcance na rede de rastreio e diagnóstico bem como condições favoráveis para dar entrada na unidade básica (CONCEIÇÃO HN, et al., 2019).

Esta discussão também oferece análise sobre os picos de diagnósticos que ocorrerem no primeiro e terceiro trimestre, em 2018, apresentando, respectivamente, 1601 e 1794 casos, diferente do segundo trimestre que apresentou 1113 casos. O diagnóstico de sífilis gestacional é mais prevalente nos estágios primário e latente da doença, em que os casos de 2010 a 2019 ultrapassam os 6000 diagnósticos em ambos os estágios, atingindo as porcentagens de $41,4 \%$ e $42,6 \%$, respectivamente.

Além disso, percebe-se que os diagnósticos aumentaram ao longo dos anos, atingindo o auge em 2018. Obtiveram-se nesse ano, portanto, a maior incidência de casos de sífilis gestacional em Minas Gerais, subdivididos em 1646 diagnósticos de sífilis primária, 276 diagnósticos de sífilis secundária, 312 diagnósticos da fase terciária da doença e 1269 casos diagnosticados como sífilis latente. Em 2019, os casos diminuíram significantemente, alcançando números reduzidos em até 63,9\% quando comparados com os do ano anterior, como no caso da sífilis primária (Tabela 5).

Tabela 5 - Distribuição absoluta (n) e percentual (\%) de casos de gestantes com sífilis segundo classificação clínica por ano de diagnóstico.

\begin{tabular}{ccccc}
\hline Ano & $\begin{array}{c}\text { Sífilis Primária } \\
\mathbf{n}(\%)\end{array}$ & $\begin{array}{c}\text { Sífilis Secundária } \\
\mathbf{n}(\%)\end{array}$ & $\begin{array}{c}\text { Sífilis Terciária } \\
\mathbf{n}(\%)\end{array}$ & $\begin{array}{c}\text { Sífilis Latente } \\
\mathbf{n}(\%)\end{array}$ \\
\hline $\mathbf{2 0 1 0}$ & $121(33,4)$ & $25(6,9)$ & $13(3,6)$ & $160(11,9)$ \\
$\mathbf{2 0 1 1}$ & $188(34,2)$ & $51(9,3)$ & $24(4,4)$ & $227(10,9)$ \\
$\mathbf{2 0 1 2}$ & $258(29,6)$ & $74(8,5)$ & $43(4,9)$ & $419(8,8)$ \\
$\mathbf{2 0 1 3}$ & $367(32,3)$ & $79(6,9)$ & $63(5,5)$ & $549(7)$ \\
$\mathbf{2 0 1 4}$ & $633(37,3)$ & $111(6,5)$ & $103(6,1)$ & $698(9)$ \\
$\mathbf{2 0 1 5}$ & $822(34,1)$ & $189(7,8)$ & $130(5,4)$ & $1010(10,7)$ \\
$\mathbf{2 0 1 6}$ & $830(31,9)$ & $240(9,2)$ & $140(5,4)$ & $1014(14,6)$ \\
$\mathbf{2 0 1 7}$ & $1296(35,3)$ & $264(7,2)$ & $221(6)$ & $1124(20,8)$ \\
$\mathbf{2 0 1 8}$ & $1646(33,9)$ & $276(5,7)$ & $312(6,4)$ & $1269(25,7)$ \\
$\mathbf{2 0 1 9}$ & $593(31,4)$ & $106(5,6)$ & $124(6,6)$ & $479(31,1)$ \\
\hline Total & $6754(41,4)$ & $1415(8,6)$ & $1173(7,2)$ & $6949(42,6)$ \\
\hline
\end{tabular}

Notas: (1) Dados até 30/06/2019; (2) Dados preliminares para os últimos 5 anos.

Fonte: Santos SS, et al., 2021; dados extraídos de MS/SVS/DCCI - Departamento de Doenças de Condições Crônicas e Infecções Sexualmente Transmissíveis.

O número crescente de diagnósticos se justifica devido a orientação do Ministério da Saúde no qual determina que a realização de teste rápido para sífilis seja ministrada no $1^{\circ}$ trimestre e se refaça no $3^{\circ}$, além da realização deste no momento da internação para o parto. Tal ação se priorizou nesta fase pois a relação do feto e seu acometimento dependerá do tempo de infecção e tempo de gestação. A fase entre a $28^{\mathrm{a}}$ e $30^{\mathrm{a}}$ semana é importante pois ainda é possível realizar o tratamento na fase intrauterina e ainda dentro da possibilidade de tratar a mãe com prazo de 30 dias antes do parto (LACERDA ED, et al., 2017). 
A distribuição dos diagnósticos de sífilis vai de encontro à relatada por Freitas GM, et al. (2019), que mostra que a sífilis terciária obteve maior frequência entre 2010 e 2017 em Alfenas-MG. Outro estudo realizado no Ceará também mostra que a classificação terciária da doença prevaleceu, representada por $75 \%$ da amostra no período de 2012 a 2017. Além disso, o tratamento na fase primária e secundária é mais simples e há menor número de abandono quando comparado com o tratamento realizado na fase terciária e latente, nos quais muitas vezes não é finalizado (MARQUES JVC, et al., 2018). Tendo isso em vista, o tratamento para os casos de sífilis em Minas Gerais, teoricamente, seria mais resolutivo, considerando a adesão dos pacientes, quando comparado com a terapêutica no Ceará.

Portanto, considerando que o ápice da transmissão da sífilis ocorre nas fases primária e secundária da doença, o correto rastreamento e diagnóstico pelos profissionais da saúde são de suma importância para tratamento e inibição da transmissão, uma vez que o tratamento seria mais eficaz (FREITAS GM, et al., 2019). Considerando o tratamento para sífilis prescrito por diagnóstico, percebe-se a prevalência de casos tratados em Minas Gerais com penicilina de 2015 a 2018. Entretanto, o número de casos em que o tratamento não foi realizado também cresce ao longo dos anos, atingindo $8,2 \%$ dos prescritos (Tabela 6 ).

Tabela 6 - Distribuição absoluta (n) e percentual (\%) de casos de gestantes com sífilis segundo esquema de tratamento prescrito por ano de diagnóstico.

\begin{tabular}{lcccc}
\hline Ano & $\begin{array}{c}\text { Penicilina } \\
\mathbf{n}(\%)\end{array}$ & $\begin{array}{c}\text { Outro Esquema } \\
\mathbf{n}(\%)\end{array}$ & $\begin{array}{c}\text { Não Realizado } \\
\mathbf{n}(\%)\end{array}$ & $\begin{array}{c}\text { Ignorado } \\
\mathbf{n}(\%)\end{array}$ \\
\hline $\mathbf{2 0 1 5}$ & $1.918(79,6)$ & $136(5,6)$ & $221(9,2)$ & $135(5,6)$ \\
$\mathbf{2 0 1 6}$ & $2.109(81)$ & $115(4,4)$ & $219(8,4)$ & $162(6,2)$ \\
$\mathbf{2 0 1 7}$ & $3.085(84,1)$ & $141(3,8)$ & $328(8,9)$ & $113(3,1)$ \\
$\mathbf{2 0 1 8}$ & $4.146(85,5)$ & $185(3,8)$ & $354(7,3)$ & $166(3,4)$ \\
\hline Total & $1.1258(83,1)$ & $577(4,2)$ & $1.122(8,2)$ & $576(4,2)$ \\
\hline
\end{tabular}

Notas: (1) Dados até 30/06/2019; (2) Dados preliminares para os últimos 5 anos.

Fonte: Santos SS, et al., 2021; dados extraídos de MS/SVS/DCCI - Departamento de Doenças de Condições Crônicas e Infecções Sexualmente Transmissíveis.

A prevalência do tratamento por penicilina segue a preconização do Ministério da Saúde para o esquema de tratamento de sífilis: utilização de penicilina como medicamento de escolha. A penicilina é eficaz no tratamento da gestante e na prevenção da transmissão vertical e sua posologia depende do estágio da doença. É primordial promover a saúde para a mãe e para o concepto, dessa maneira destaca-se a urgência do diagnóstico, já que no primeiro trimestre de gravidez o tratamento correto da mãe é eficaz e evita a infecção do filho, prevenindo sequelas futuras (FREITAS GM, 2019; CAMPOS CO, 2020).

Observa-se, também, que a quantidade de casos tratados, tanto com penicilina quanto com outros esquemas, aumenta ao longo dos anos. Nesse aspecto, destaca-se o medicamento Ceftriaxone, utilizado em situações específicas quando há desabastecimento de penicilina (RIBEIRO MD, 2020). Em relação ao aumento do número de casos não tratados, esse achado condiz com o estudo no sul de Minas Gerais, em 2017, no qual a porcentagem de tratamentos não realizados é bem próxima: $8,5 \%$ dos casos diagnosticados (FREITAS GM, 2019; RIBEIRO MD, 2020).

Ademais, o número de casos diagnosticados em gestantes a partir da triagem para sífilis aumentou desde 2014 no Brasil com o uso do teste rápido nas consultas pré-natal. Esse feito contribuiu para o aumento da incidência e notificação da doença, o que justifica o aumento do número de casos diagnosticados. Além disso, a triagem rápida para sífilis colaborou para detecção de pacientes tanto sintomáticos quanto assintomáticos. Essa informação esclarece o crescente número de casos diagnosticados, porém não tratados, já que o número de notificações de diagnósticos em pacientes assintomáticos elevou (FIGUEIREDO DCMM, 2020). 


\section{CONCLUSÃO}

Evidencia-se um crescimento significativo na incidência da Sífilis na gestação ao longo dos anos no estado de Minas Gerais, caracterizado por mulheres adultas jovens, pardas e que têm ensino fundamental incompleto. O diagnóstico da sífilis na gestação ocorre de forma tardia, no terceiro trimestre, tendo como tratamento, na maioria dos casos, a penicilina. O preenchimento das fichas de notificação da doença que vem ocorrendo de forma mais eficiente ao longo dos anos, permitiu analisar de maneira eficaz, sua epidemiologia no estado. Isso reforça a importância e a necessidade de manter profissionais de saúde e estudantes treinados e atualizados, a fim de garantir detecção precoce, tratamento oportuno e acompanhamento efetivo da sífilis gestacional.

\section{REFERÊNCIAS}

1. ANDRADE HS, et al. Caracterização epidemiológica dos casos de sífilis em mulheres. Ciência \& Saúde, 2019; 12(1): $1-5$.

2. ARAÚJO AGR, et al. Estudo epidemiológico dos casos de sífilis em gestantes em uma cidade do norte de Minas Gerais. Revista Eletrônica Acervo Saúde, 2018; 11(3): 1-9.

3. BARBOSA DFR, et al. Perfil epidemiológico da sífilis congênita em gestantes no município de Maceió. Revista Eletrônica Acervo Saúde, 2020; 12(11): 1-9.

4. BRASIL. Ministério da Saúde. Teste rápido de HIV e Sífilis na atenção básica. 2017.

5. BRASIL. Protocolos assistenciais de obstetrícia da maternidade climério de oliveira: Sífilis na Gestação. Ministério da Educação. EBSERH. Hospitais Universitários Ferderais. Maternidade Climério de Oliveira - MCO-Universidade Federal da Bahia - UFBA. Salvador: UFBA, c.11, 2018.

6. CABRAL BTV, et al. Sífilis em gestante e sífilis congênita: um estudo retrospectivo. Revista Ciência Plural, 2017; 3 : $32-44$

7. CÂMARA LS, et al. Perfil epidemiológico das gestantes com sífilis no Rio de Janeiro. Research, Society and Development, 2020; 9(10): 1 -14.

8. CAMPOS CO, CAMPOS CO. Abordagem diagnóstica e terapêutica da sífilis gestacional e congênita: revisão narrativa. Revista Eletrônica Acervo Saúde, 2020; 53: 1-7.

9. CÂNDIDO SD, et al. A epidemiologia da sífilis e a reincidência dos casos na cidade de São Lourenço, MG. Revista Saúde em Foco, Teresina, 2019; 11: 239-251.

10. CONCEIÇÃO HN, et al. Análise epidemiológica e espacial dos casos de sífilis gestacional e congênita. Saúde Debate, 2019; 43(123): 1145-1158.

11. CRUZ VC. Sífilis Congênita: análise espacial e aspectos epidemiológicos das mães. Dissertação (Mestrado em Enfermagem) - Universidade Federal de Sergipe, Aracajú, 2019; 81p.

12. FIGUEIREDO DCMM, et al. Relação entre oferta de diagnóstico e tratamento da sífilis na atenção básica sobre a incidência de sífilis gestacional e congênita. Cad. Saúde Pública, 2020; 36(3): 1-12.

13. FREITAS GM, et al. Notificação da sífilis adquirida em uma superintendência regional de saúde do sul de minas gerais. Cogitare Enfermagem, 2019; 24: 1-12.

14. GOMES NS, et al. Produção Científica na área da saúde sobre sífilis gestacional: revisão narrativa. SANARE (Sobral, Online), 2020; 19(1): 113-120.

15. JÚNIOR WVO, et al. Investigação de sífilis no município de Lagoa da Prata - MG: Análise da incidência de Sífilis congênita e efetividade do tratamento de gestantes portadoras de Sífilis. Revista Conexão Ciência, 2020; 15(2): 51 61.

16. LACERDA ED, et al. Controle da sífilis na gestação: uma abordagem sobre a assistência de enfermagem. Temas em Saúde, 2017; 17(2): 226-235.

17. MACÊDO VC, et al. Sífilis na gestação: barreiras na assistência pré-natal para o controle da transmissão vertical. Cad. Saúde Colet., 2020; 28(4): 518-528.

18. MACHIO-LIMA T, et al. Perfil epidemiológico de pacientes com sífilis congênita e gestacional em um município do Estado de São Paulo, Brasil. Rev. Bras. Saúde Mater. Infant., 2019; 19(4): 873-880.

19. MARQUES JVS, et al. Perfil epidemiológico da sífilis gestacional: clínica e evolução de 2012 a 2017. Sanare, 2018; 17(02): 13-20.

20. MORAIS TR, et al. Interseccionalidades em Saúde: Predomínio de Sífilis Gestacional em Mulheres Negras e Pardas no Brasil. Id on Line Rev. Mult. Psic., 2019; 13(45): 670-679.

21. PADOVANI C, et al. Sífilis na gestação: associação das características maternas e perinatais em região do sul do Brasil. Rev. Latino-Am. Enfermagem, 2018; 26: 1-10.

22. PEREIRA AL, et al. Impacto do grau de escolaridade e idade no diagnóstico tardio de sífilis em gestantes. Revista Feminina, 2020; 48(9): 563-567.

23. RIBEIRO MD, et al. Análise retrospectiva da evolução clínica dos casos de sífilis congênita tratados com ceftriaxone ou penicilina nos anos de 2016 até junho de 2018 no ambulatório de referência no município de Teresópolis. Revista da Jopic, 2020; 3(6), 2525-7293.

24. SABACK MC, et al. Perfil epidemiológico da sífilis gestacional e congênita na Maternidade Ana Braga - Manaus, Amazonas. Revista Eletrônica Acervo Saúde, 2018; 11(5): 1-9.

25. SANTOS VF, et al. Perfil epidemiológico de los casos de sífilis en embarazadas en una maternidad pública. Cultura de los Cuidados, 2019; 23(54): 396-406.

26. SOUZA RR, et al. Perfil de Casos Notificados de Sífilis Congênita no Estado de Goiás entre 2015 a 2018. Braz. J. of Develop., 2020; 6(7): 48715-48725. 